\title{
Detection of coherent vorticity structures using time-scale resolved acoustic spectroscopy
}

\author{
Christophe Baudet, Olivier Michel, \\ Laboratoire de Physique (URA 1325 CNRS), École Normale Supérieure de Lyon, \\ 46 allée d'Italie, 69364 Lyon Cedex 07, France \\ tel : (33) 472728378 - fax : (33) 472728080 \\ e-mail : baudet@physique.ens-lyon.fr, omichel@physique.ens-lyon.fr \\ William J. Williams \\ Department of Electrical Engineering and Computer Science, \\ University of Michigan, Ann Arbor, MI 48109-2122, USA \\ e-mail : wjw@gabor.eecs.umich.edu
}

January 15,2014

Submitted to : Physica D

\section{PACS}

47.32. Vortex dynamics

47.27.Gs Turbulent Flows - Isotropic turbulence

43.60. Acoustic signal processing (43.60.Rw, 43.60.Gk)

ENSLYON • PHYSIQUE 


\begin{abstract}
It seems widely accepted by the turbulence community that the intermittency observed in fully turbulent flows is closely related to the existence of intense vorticity events, localized in time and space, also known as coherent structures. We describe here an experimental technique based on the acoustic scattering phenomenon allowing the direct probing of the vorticity field in a turbulent flow. In addition, as in any scattering experiment, the information is in the Fourier domain : the scattered pressure signal is a direct image of the time evolution of a well specified spatial Fourier mode of the vorticity field. Using time-frequency distributions, recently introduced in signal analysis theory, for the analysis of the scattered acoustic signals, we show how the legibility of these signals is significantly improved (time resolved spectroscopy). The method is illustrated on data extracted from a highly turbulent jet flow : discrete vorticity events are clearly evidenced. The definition of a generalized time-scale correlation function allows the measurement of the spatial correlation length of these events and reveals a time continuous transfer from large scale towards smaller scales (turbulent cascade). We claim that the recourse to time-frequency distributions leads to an operational definition of coherent structures associated with phase stationarity in the time-frequency plane.
\end{abstract}


Intermittency [1] is usually revealed by analyzing the statistical properties of the flow deduced from single point measurements (e.g. the time record of the flow velocity at one point obtained by hot wire anemometry). In the past few years, the turbulence community has reached a consensus about an acceptable definition of intermittency, this latter being defined as deviations (or corrections) from the power law scaling of the longitudinal velocity structure functions in the inertial range: $S_{p}(l)=\left\langle\left(\delta v_{\|}(l)\right)^{p}\right\rangle \propto l^{\zeta_{p}}$ initially proposed by Kolmogorov in 1941 (K41 theory). Indeed, the K41 theory predicts that $\zeta_{p}=\frac{p}{3}$, whereas clear deviations from this linear law are experimentally observed [2]. Recent results suggest that intermittency is closely related to the presence of localized (in time and space) vortices in the turbulent flow. Such objects, presumably with a filamentary shape, have been observed in some experiments [3, 4] and numerical simulations [5, 6]. Theoretical models [7] assuming a hierarchy of coherent structures in the form of vortex filaments, predict a relation for the $\zeta_{p}=f(p)$ in very good agreement with experimental data. However, a clear definition of coherent vorticity structures is still lacking.

There exists a vast amount of theoretical work dealing with the acoustic scattering phenomenon by velocity fields. Most of it was initiated nearly fifty years ago and started with the papers authored by A.M. Obukhov [8], R.H. Kraichnan [9], Chu and Kovasznay [10], to mention but a few. In a more recent paper, F. Lund 11] has established, under reasonable assumptions, a linear relation between the scattered amplitude of a plane acoustic wave incident on a turbulent flow and the spatial Fourier transform of the vorticity field. The purpose of this paper is to demonstrate how one can take advantage of the spectral nature of the measurement process, in an acoustic scattering experiment, for the detection of spatially and temporally localized vorticity events. Indeed, there are numerous examples in physics of disordered media, where scattering techniques (e.g. light and neutron scattering in condensed matter physics) pro- 
vide useful and pertinent informations about the statistical properties of these systems.

This experimental work aims at introducing, on experimental basis, a new method for investigating turbulent flows, serving as an alternate to usual single point measurements like hot wire anemometry. We place the emphasis on some experimental evidence of the existence of coherent and localized (i.e. intermittent in both space and time) structures. Using a dual channel setup (acoustic interferometer), we are able to experimentally demonstrate the effectiveness of the length scale selection (through the selection of a well defined spatial scattering wave vector) involved in acoustic scattering measurements. Further, as intermittency implies non stationarity of physical quantities (think about a vortex as being localized in both time and frequency domains, appearing or disappearing within the analyzed volume), the second part of this paper focuses on non stationary spectral analysis. The proposed approach is based on the recently introduced [12] Reduced Interference time-frequency Distribution (RID), of which the properties are briefly discussed. RID analysis is then applied to signals from an experiment of acoustic scattering by a fully turbulent jet flow. On a single RID reprentation of the scattered pressure signals, one clearly oberves well localized energy packets at a given spatial wavevector. In order to get further insight on these objets, we compare the RID representations at two different wavevectors simultaneously recorded. This dual representation demonstrates a significant correlation of the time occurences and of the velocities (correlated phase derivatives due to correlated doppler shifts). As this time-frequency correlation decreases with the separation between the two wavevectors, we conclude that the different patterns selected at different wavectors belong to what can be defined as a coherent structure. Notice here that our approach closely matches the definition of intermittency formerly proposed by G.K. Batchelor in 1953 13: "The inference, then, is that there is an uneven distribution, in space of 
the energy associated with the large wave-number components of the turbulence, and that the higher the wave-number, the more does the associated energy tend to occur in confined regions of space (meaning that if a Fourier resolution of the velocity field within a region of activity were made, the amplitude of the component at the relevant wave-number would be found to be large, while a Fourier resolution of the velocity field within a region of quiescence would give a very small amplitude; the amplitude of the component for the field as a whole will lie somewhere between these two amplitudes)". The paper is organized as follows : part 1 is devoted to the presentation of some theoretical aspects and assumptions underlying scattering of acoustic waves by a turbulent flow field with a particular emphasis on the role played by the vorticity field in this interaction. In part 2 , we describe the experimental setup as well as the statistical properties of the turbulent jet flow under investigation. The data acquisition procedure and the preprocessing of the recorded data are detailed in part 3 . Part 4 deals with some theoretical issues of time frequency analysis and the role of the ambiguity function for introducing the RID. Finally, experimental results are displayed and discussed in part 5 .

\section{Acoustic scattering by a turbulent flow}

As quoted by Nelkin [14], vorticity is intrinsically a non-local quantity, and therefore it can hardly be measured by using local probes (e.g. hot wire anemometers) [16]. Although vorticity could "conceptually" be measured by local probes, the main problem resides in that one needs at least two probes, separated by a finite distance in order to compute the gradients involved by the curl operator. From an experimental point of view the situation is even worse: at least nine probes are needed to reconstruct the three components of the vorticity, within the appropriate Taylor Hypothesis (see [16]). Since these probes have a finite extension and finite time response, the computation of the gradients at the smallest scale of the turbulent flow becomes problematic [17, 18]. It is worth 
noticing here that an alternate experimental has been proposed by Noullez \& al. 15] in order to probe directly the spatial transverse velocity increments in a turbulent flow.

Taking these latter remarks into account, one can find several theoretical [8, 9, 10], experimental [20, 21, 22, 23, 24, 26] and numerical works 19] addressing the direct measurement of the vorticity field using acoustic scattering. As in any scattering experiment, one expects to be sensitive to the spatial Fourier distribution of the scatterers (as the amplitude of the scattered wave is expressed in terms of some coherent average on "secondary sources") over a finite volume defined as being the cross section of the acoustic beams (incident and scattered). In a recent paper, Lund et al. 11] derive a linear relation between the Fourier transform of the scattered ultrasound amplitude and the space-time Fourier transform of the vorticity field. In analogy with the more usual light scattering phenomenon, the physical mechanism at the origin of acoustic scattering by vorticity can be thought of as follows : an acoustic wave impinging on a vorticity distribution induces fluctuations of the vorticity at the incoming sound frequency (by virtue of the Kelvin circulation theorem). Each scatterer (vortex) acting as a secondary source will, in turn, radiate a sound wave. The coherent average (taking into account the relative positions of the individual vorticity elements) over the scatterers distribution results in the emission, outside the vorticity domain, of a scattered acoustic wave. It is important to emphasize here that many vortices interact simultaneously with the wave front. Such interactions have been numerically investigated in 19 in the case where a unique vortex is present. In a scattering process, one is sensitive to spatial details of the scatterers distribution at a typical length scale measured by the wavelength. Note that whereas a light scattering process is usually linear, the acoustic scattering phenomenon depicted here stems from the non-linear term of the Navier-Stokes equation, and requires much explanations and details com- 
putations that are beyond the scope of this paper. In particular, one can find in [11 a clear explanation of the respective contributions to the acoustic scattering cross-section of the vorticity field on one hand, and of the irrotational velocity field, induced by the vorticity, on the other.

Using a Born approximation, Lund et al. obtain the following linear relation between the scattered acoustic pressure amplitude and the spatial Fourier transform of the vorticity field:

$$
\frac{p_{\text {scat }}(\nu)}{p_{\text {inc }}}=\pi^{2} i \frac{-\cos \left(\theta_{s}\right)}{1-\cos \left(\theta_{s}\right)} \frac{\nu e^{i \nu D / c}}{c^{2} D}(\vec{n} \wedge \vec{r}) \cdot \vec{\Omega}\left(\vec{q}_{s c a t}, \nu-\nu_{o}\right)
$$

where $\wedge$ and "." stand for the vector product and the scalar product respectively, and

$$
\vec{q}_{s c a t}=\frac{2 \pi}{c}\left(\nu \vec{r}-\nu_{o} \vec{n}\right) \simeq \frac{4 \pi \nu_{o}}{c} \sin \left(\frac{\theta_{s}}{2}\right) \frac{\vec{r}-\vec{n}}{|\vec{r}-\vec{n}|} \text { for } \nu \simeq \nu_{o}
$$

In these formula,

- $\vec{q}_{\text {scat }}$ is the scattering wave vector

- $\vec{n}$ and $\vec{r}$ are the impinging and scattered wave directions, respectively

- $\nu_{o}$ is the frequency of the incoming sound

- $\nu$ is the frequency of the scattered sound

- $\theta_{s}$ is the scattering angle

- $p_{i n c}$ stands for the pressure amplitude of the incoming sound wave (assumed to be plane and monochromatic)

- $p_{\text {scat }}$ is the amplitude of the scattered sound wave

- $D$ and $c$ stand for the acoustical path between the measurement area and the detector, and the sound velocity respectively.

- $\vec{n}$ and $\vec{r}$ are the unit vectors in the direction of the probing and detected sound waves 
It is important to emphasize here that among the hypothesis used in [11] the characteristic time of any process within the flow has to be very large compared with the period of the analyzing sound wave. This latter remark implies that $\nu \simeq \nu_{o}$ and that this method is relevant only for flows with low Mach number. Equation (11) shows that, using acoustic scattering, it is possible to probe one component (perpendicular to the scattering plane defined by the vectors $\vec{n}$ and $\vec{r})$ at a chosen length scale through the selection of a known scattering wave vector $\vec{q}_{s c a t}$.

In a previous similar experiment [24] (see also [25]) performed on the regular vortex street behind a cylinder at low Reynolds numbers, we have demonstrated that the scattering cross section is indeed strongly dependent on the direction of the vorticity field accordingly to Equation (1) of the present paper. This latter experiment evidences the existence of direct interactions between vorticity and acoustic waves : among the nine components of the stress tensor only one vector can be defined without ambiguity, i.e. the vorticity (antisymmetric part of the tensor). Furthermore, a nice feature of this method lies in the fact that it is a non perturbating technique.

\section{Experimental setup}

The acoustic waves are produced and detected by Sell-type transducers (see e.g. 29]), the dimensions of which are $15 \mathrm{~cm} \times 15 \mathrm{~cm}$. The advantage of dealing with such large transducers is to minimize diffraction effects and therefore to enable a sharp selectivity of the scattering wave vectors ( $\vec{q}_{\text {scat }}$ defined above), both in modulus and direction [24]. As shown on figure (1), the direction of $\vec{q}_{s c a t}$ is aligned with the mean-flow velocity in order to maximize the Doppler shift. The magnitude of $\vec{q}_{s c a t}$, and thus the probed length-scale in the flow, is then selected by tuning the incoming sound frequency $\nu_{o}$, the scattering angle being held fixed. Indeed, for practical purposes, it is much simpler to hold the 
geometry of the experimental setup constant. Therefore, $\theta_{s}$ is constant and $\vec{q}_{\text {scat }}$ has constant direction. The scattering angle $\theta_{s}$ was set to 60 degrees, as a trade-off between optimizing the sensitivity of the measurements and limiting the spurious effects induced by diffraction side-lobes 24 . It can then be seen from (11), that the Fourier transform (FT) of the scattered pressure signal is proportional to the band-pass spatial frequency filtered vorticity field. The central frequency of the latter filter is $q_{s c a t}\left(\nu_{o}\right)=\left\|\vec{q}_{s c a t}\left(\nu_{o}\right)\right\|$. Its width $(\Delta q)$ can be obtained from the diffraction pattern of the Sell transducers [24. A rough estimate of $\Delta q$ is given by differentiating $\vec{q}_{s c a t}$ with respect to $\theta_{s}$, which leads to $\Delta q=\frac{2 \pi \nu_{o}}{c} \cos \left(\theta_{s} / 2\right) \cdot 2 \Delta \theta$, where $\Delta \theta$ is given by a diffraction ansatz: $\Delta \theta \simeq \frac{c}{\nu_{o} L}, L$ being the characteristic size of the transducer [30]. The volume which is analyzed is defined by the cross section of the incident beam and the main lobe of the receiver (antenna beam). Its dimensions in our experimental setup were of the same order of magnitude (a bit larger) than the transducers dimensions.

The flow which we investigate in this paper, consists in an axi-symmetric turbulent jet flow $\left(R_{\lambda} \simeq 600\right)$. emerging from a circular nozzle of diameter $5 \mathrm{~cm}$, at a high velocity (about $60 \mathrm{~ms}^{-1}$ ). Details about our wind tunnel facility can be found elsewhere [2]. The volume of interest was set at a distance of about 50 nozzle diameters downstream ensuring that the turbulence is fully developed. A study based on classical anemometry (performed in the same area of interest) leads to the estimated following parameters : the average longitudinal velocity is about $5.3 \mathrm{~ms}^{-1}$ and the turbulence ratio is about $28 \%$ [26]. The third order structure function exhibits a clear power law scaling over 1.5 decades (from the Taylor scale $\lambda=6 \mathrm{~mm}$ up to the integral length scale $l_{o}=.25 \mathrm{~m}$ ), with an exponent close to 1 in accordance to the Kolmogorov four-fifths law [1]. From this inertial range scaling, we deduce the value of the mean energy dissipation per unit mass $\epsilon=13 \mathrm{~m}^{2} \mathrm{~s}^{-3}$, the Kolmogorov micro scale $\eta=131 \mu \mathrm{m}$ and the large eddy turnover time $t_{o}=168 \mathrm{~ms}$. The analyzing sound frequency can be varied 
from $5 \mathrm{kHz}$ up to $200 \mathrm{kHz}$ (where sound attenuation effects cannot be neglected). Thus we are able to probe a large part of the inertial range, as well as length scales belonging to the beginning of the dissipation range [26]. However in this paper, we shall restrict our analysis to a single length scale pertaining to the inertial range $\left(\frac{q_{s c a t}}{2 \pi}=0.6 \mathrm{~cm}^{-1}\right.$ corresponding to a typical length scale of 1.7 $\mathrm{cm})$.

\section{Data acquisition and pre-processing}

\subsection{Single channel measurements}

An experiment consists in measuring and recording time series of the acoustic pressure signal received on a Sell-type transducer (see above). The emitted sound wave can be considered as being a monochromatic plane wave (if diffraction effects are neglected at first sight). The sampling device (HP3565) has 16 bit resolution and its sampling frequency is $262144 \mathrm{~Hz}$. Each recording channel is provided with its own local oscillator (tuned at $\nu_{o}$ ), thus enabling a numerical heterodyne detection of the scattered signal around the frequency of the impinging sound wave. Note also that, in order to reduce phase noise along the whole measurement line, the waveform generators (sound emission), the sampling clock and the local oscillators (demodulation) are locked on the same $10 \mathrm{MHz}$ master clock. Two time series associated with synchronous phase and quadrature signals are recorded at a rate of 8192 samples per second, from which the analytical complex signal is computed (characterized by a phase and an amplitude). Indeed, thanks to the linearity of our ultrasonic detectors, the phase information of the scattered pressure wave is preserved (the phase reference being that of the incoming sound wave).

As indicated by equation (值) (expressing a convolution in the time Fourier domain), the scattered pressure signal is obtained as a time modulation of the incoming pressure signal (carrier wave) by the low frequency signal associated with the vorticity of the flow under investigation (for small enough Mach num- 
bers). One thus expects a narrow band scattered signal with frequencies close to the incoming sound frequency $\nu_{o}$ (see figure 2 a). Then, the purpose of the above mentioned heterodyne detection is to significantly reduce the amount of stored data. Equations (1, 2) lead (for $\nu \simeq \nu_{o}$ ) to express the Fourier transform of the detected pressure signal as

$$
p_{\text {scat }}(\nu) \propto \frac{\cos \left(\theta_{s}\right) \cos \left(\frac{\theta_{s}}{2}\right)}{q_{\text {scat }}} \vec{\Omega}_{\perp}\left(q_{\text {scat }}, \nu-\nu_{o}\right)
$$

where $q_{\text {scat }}$ and $\vec{\Omega}_{\perp}$ stand for the magnitude of the scattering wave-vector and the vorticity component orthogonal to the scattering plane, respectively. We have represented on Figure 2 (a) a typical averaged power spectral density of the scattered pressure signal (after demodulation) obtained in the turbulent jet flow. Notice that in this figure and throughout this paper, the frequency axis represents the frequency shift with respect to the incoming sound frequency $\nu_{o}$. The spectrum is clearly non-symmetric with respect to the null frequency. This latter fact is a simple consequence of the advection of the vorticity field by the flow velocity and to our decision to align the scattering wave vector with the direction of the mean flow velocity $\vec{V}_{\text {mean }}$. Indeed, the scattering of waves by a moving structure gives rise to a frequency shift $\Delta \nu$ as a consequence of a Doppler effect [31] :

$$
\Delta \nu=\nu-\nu_{o}=\frac{1}{2 \pi} \vec{q}_{s c a t} \cdot \vec{V}_{\text {mean }}
$$

Notice that this latter relation can be obtained by applying a Galilean transform in the real space followed by a double Fourier transform on the space and time variables.

From (3) one expects a divergence of the scattered signal around $\theta_{s}=0$, for which $q_{\text {scat }}=0$ and thus $\Delta \nu=0$. Indeed, the existence of direct paths between the emitter and receiver (associated to diffraction effects due to the finite size of the transducers) results in the presence of a very strong peak in the spectrum $p_{\text {scat }}(\Delta \nu)$ around $\Delta \nu=0$. From (何), we are able to relate $q_{\text {scat }}$ to $\Delta \nu$, and thus 
get the following expression for the received acoustic signal

$$
p_{\text {scat }}\left(\nu_{0}+\Delta \nu\right) \propto \frac{1}{\Delta \nu} \vec{\Omega}_{\perp}\left(q_{\text {scat }}, \Delta \nu\right)
$$

Bearing in mind that the scattered pressure signal is recorded after a numerical heterodyning procedure that shifts its analytical frequency spectrum around the null frequency (the frequency of local oscillator in the lock-in is set to $\nu_{o}$ ), one gets

$$
P_{\text {scat }}(\Delta \nu) \propto \frac{1}{\Delta \nu} \vec{\Omega}_{\perp}\left(q_{\text {scat }}, \Delta \nu\right)
$$

where $P_{\text {scat }}$ stand for the scattered pressure recorded signal. Taking the inverse FT of equation (6) leads to

$$
P_{\text {scat }_{r e c}}(t)=\int_{-\infty}^{+\infty} \vec{\Omega}_{\perp}\left(q_{\text {scat }}, t\right) d t
$$

The preceding equation expresses the recorded scattered signal (after heterodyning) as being the integral of $\vec{\Omega}_{\perp}\left(q_{\text {scat }}, t\right)$ with respect to the time variable As a consequence, the recorded data must be differentiated to give a correct

representation of $\vec{\Omega}_{\perp}\left(q_{\text {scat }}, t\right)$. It is important to notice here that the latter differentiation must be performed at a low frequency, since the heterodyne detection has already been applied. The averaged power density spectrum (PDS) of $p_{\text {scat }}(t)$ recorded for the jet flow (see preceding section) and $\nu_{o}=32 \mathrm{kHz}$, is shown on figure (2a). Figure (2) b) illustrates the efficiency of the difference filter applied to the time series for extracting a PDS proportional to $\vec{\Omega}_{\perp}\left(q_{\text {scat }}, \Delta \nu\right)$. It is worth noticing at this point that this differentiation ansatz is nothing else than a transposition of the Taylor hypothesis (heavily used in single point measurement). Actually, it suffers from the same limitations with regards to the level of turbulence.However, as explained below, these limitations can be overcome by the resort to the time-frequency distributions.

\subsection{Two-channels measurements}

According to (2), a given spatial spectral component of the vorticity field may be probed by using different sets $\left(\theta_{s}, \nu_{0}\right)$, associated with a couple of trans- 
ducers (one emitter, one receiver) which will be referred to as a channel in the rest of the paper. The acoustical paths corresponding to the null scattering angle for which divergence occurs (thus explaining the presence of a strong peak at zero frequency shift, see (何) is then different for each set of ultrasonic emitter-receiver. As a consequence, it is expected that the parts of the signals corresponding to the null scattering angle, recorded on each channel are mutually non coherent. Following the same reasoning, it is expected to find a high degree of coherence between parts of signals that are due to scattering effects from the same measurement area (cf preceding sections), provided that both channels are tuned to probe the same scattering wave-vector:

$$
\frac{4 \pi \nu_{0,1}}{c} \sin \left(\frac{\theta_{s, 1}}{2}\right)=\frac{4 \pi \nu_{0,2}}{c} \sin \left(\frac{\theta_{s, 2}}{2}\right)
$$

where $\nu_{0, i}, \theta_{s, i}$ stands for the ultrasound frequency and scattering angle associated with channel $i$.

The additional hypothesis in the 2-channel measurement scheme, is that the measurement areas defined by the 2 sets of transducers are actually identical. This point is crucial in our experiment, as any mismatch in the measurement areas would preclude any possibility to compare scattering signals, which would then be associated to different spatial distributions. The setting of the experiment is derived by carefully aligning the transducers with laser diode rays. Then, the lengths of the acoustical paths for each channels are set to the same value (up to a precision of $1 \mathrm{~mm}$ ) 1 , in order to insure equivalent propagations delays on both channels. This is achieved by checking the phase of the cross channels inter-spectrum estimates, and shifting the transducers along its main diffraction axis accordingly: for null propagation delay, the slope of the phase with respect to frequency must be zero, as group delays are expected to be identical on both channels.

Figure (3) illustrates the fact that the divergences at zero-frequency shifts, mea-

\footnotetext{
${ }^{1}$ The propagation time of acoustic waves over this distance is about $3 \cdot 10^{-6} s$, i.e. much smaller that the sampling period.
} 
sured on two channels verifying (8) are mutually non coherent, whereas the part of the spectrum corresponding to the scattered waves from the same measurement area (Doppler shifted) are highly similar (high cross-coherence values). Furthermore, the values obtained for the cross-coherence do not depend on whether the differentiation (see preceding paragraph) was performed, as the same linear filter (time differentiation) is applied to both channels. This latter observation illustrates the pertinence of the differentiation for extracting the interesting part (i.e. the signature of the vorticity) in the recorded signal. The phase of the cross-channels inter-spectrum is shown on the last plot of figure (3): one sees that the phase slope takes low values within the frequency domain where the spectrum of scattered signals is significant.

So far, we have dealt with second order statistical properties of the turbulent flow, and considered long enough records, to insure that the stationary hypothesis was correctly met. However, the spectrum of the recorded time-series corresponding to one spatial frequency component of the vorticity field, turns out to have an important width (Equivalent $10 \mathrm{~dB}$ bandwidth $=400 \mathrm{~Hz}$ ). This may be ascribed to either fluctuations of the advection velocity (thus leading to fluctuations of the Doppler shift), or to finite duration of vorticity structures. In this latter case, one then expects to record "sine-wave trains' of finite duration. Notice that both effects are likely to occur simultaneously. Therefore, one is naturally led to look for an analyzing tool allowing a joint representation in time (in order to access "vortex life time") and frequency (for evidencing Doppler shifts, or equivalently, advection velocity fluctuations).

\section{Time-frequency analysis}

When finite duration events of vorticity are present, the scattered acoustic signal will exhibit a time-varying spectrum, as the frequency of the measured pressure signal drops from $\nu_{0}$ to $\nu_{0}+\delta \nu$ when a vorticity event exists, that has energy in the spatial Fourier domain at the wave-vector $\vec{q}_{s c a t}$. Due to the randomness 
of the velocity field which advects the vorticity field, the finer details of the time dynamics of individual events are wiped out when average spectra of the scattered signals are computed. Indeed, on the average, the scattered spectrum has a Gaussian shape reflecting the statistical properties of the flow velocity [26. Notice that an infinitely thin and localized vortex (theoretical) would have contributions at all wave-vectors in the spatial Fourier domain.

Our aim is thus twofold : first, detecting presence/absence of such events in the recorded signal, and second, estimating the frequency shift $\Delta \nu$ at which this occurs, in order to characterize its relative velocity. We are thus faced with the need for a representation of the signal which preserve simultaneously the time and frequency information.

Temporal fluctuations of the spectral distribution can be approached by the common spectrogram analysis. Watkins [32], among others, has addressed this problem and provides examples of reasonable and unreasonable spectrogram results in the analysis of bio-acoustics signals (marine mammal sounds). However, the spectrogram involves a sliding time window which intends to capture a portion of the signal which is sufficiently restricted in time so that stationarity assumptions are approximately met. Furthermore, the presence of the window results in a time-frequency distribution (TFD) exhibiting both temporal and spectral leakage.

The Wigner-Ville distribution (WVD) avoids the problems of windowing the signal but the presence of interference terms between signal components, due to the quadratic nature of the WVD (see next paragraph) [36, 38] often precludes its applicability. Recent studies have shown that a class of smoothed WVD, the Cohen's Class of TFDs, allows to reduce the amplitude of the interference terms, while preserving covariance properties of the WVD in both the time and frequency domains. Cohen [34, 35] and Flandrin [37] have recently provided excellent reviews of $\mathrm{TF}$ analysis, including recent developments. It is worth noticing here that the above mentioned signal representations do not rule out 
the Gabor-Heinsenberg inequalities (see [36]). A brief presentation of the main issues on Cohen's class TF analysis and the key role played by the ambiguity function is given below.

In this paper, we focus on one distribution from the Cohen's class, the ReducedInterference Distribution (RID). It was first introduced by Choi and Williams [33, and has the additional feature of preserving the time and frequency support of signal components : $(R I D(t, f)$ is non zero if the signal is non zero at $t$ and has a spectral component at $f$ ). These are highly desirable properties if one wishes to infer statistical properties of the vorticity distributions from this TF analysis. The examples shown were computed with a binomial RID, which is a good discrete approximation of the continuous distribution [39].

\subsection{The WVD and Ambiguity function}

The Wigner-Ville distribution of $z(t)$ is defined as the Fourier transform $(\mathrm{F})$ of

$$
R_{z}(t, \tau)=z\left(t+\frac{\tau}{2}\right) z^{*}\left(t-\frac{\tau}{2}\right)
$$

with respect to the lag variable $\tau$.

$$
W_{z}(t, \nu)=F_{\tau}\left[z\left(t+\frac{\tau}{2}\right) z^{*}\left(t-\frac{\tau}{2}\right)\right]=F_{\tau}\left[R_{z}(t, \tau)\right]
$$

where $z(t)$ is the time signal, $z^{*}$ is its complex conjugate. The operator $F_{(.)}$ denotes the Fourier transform operator, with respect to the variable (.); this notation will be adopted in the rest of that paper. From an experimental and heuristic point of view, the effect of the operator $R_{z}(t, \tau)$ is equivalent to a local phase conjugation of the signal which enhances the phase derivative related to the Doppler shift (P. Flandrin : private communication). Similarly, but a with different physical meaning, the symmetrical ambiguity function (AF) is

\footnotetext{
${ }^{2}$ Ordinarily, the AF is defined as the FT of $R_{z}(t, \tau)$, with respect to $t$. The symmetric AF used here is defined as the inverse FT of $R_{z}(t, \tau)$, with respect to $t$. This does not induce any change in the interpretation of the AF (see 35), and is only a matter of convention. However, it allows to relate the AF to the WVD via a two-dimensional FT with respect to $t$ and $\tau$ (see equation 11 .
} 
defined as the inverse Fourier transform $\left(F^{-1}\right)$ of $R_{z}(t, \tau)$ with respect to the first variable ${ }^{3}$

$$
A_{z}(\theta, \tau)=F_{t}^{-1}\left[z\left(t+\frac{\tau}{2}\right) z^{*}\left(t-\frac{\tau}{2}\right)\right]=F_{t}^{-1}\left[R_{z}(t, \tau)\right]
$$

Thus, $W_{z}(t, \nu)$ and $A_{z}(\theta, \tau)$ are related by the two-dimensional Fourier transform

$$
W_{z}(t, \nu)=\iint A_{z}(\theta, \tau) e^{-j(t \theta+\nu \tau)} d \theta d \tau
$$

\subsection{Cohen's Class of Distributions}

Cohen's Class of distributions is defined as

$$
C_{z}(t, \nu, \phi)=\iiint e^{j((\xi-t) \theta-\nu \tau)} \phi(\theta, \tau) z\left(\xi+\frac{\tau}{2}\right) z^{*}\left(\xi-\frac{\tau}{2}\right) d \xi d \tau d \theta
$$

where $\phi(\theta, \tau)$ is the kernel of the distribution?

These relationship can be combined with Eq. (9) to show that $C_{z}(t, \nu, \phi)$ may be expressed as:

$$
C_{z}(t, \nu, \phi)=\iint \phi(\theta, \tau) A_{z}(\theta, \tau) e^{-j(t \theta+\nu \tau)} d \theta d \tau
$$

Thus, while $W_{z}(t, \nu)$ is obtained from the symmetric ambiguity function by means of two-dimensional Fourier transform, any member of Cohen's Class of distributions may be found by first multiplying its kernel $\phi(\theta, \tau)$, by the symmetric ambiguity function and then carrying out the two-dimensional Fourier transform. The spectrogram and the WVD can easily be shown to be members of Cohen's class by choice of the proper kernel in each case.

The generalized ambiguity function $\phi(\theta, \tau) A_{z}(\theta, \tau)$ is a key-concept in t-f analysis which aids one in clearly seeing the effect of the kernel in determining $C_{z}(t, \nu, \phi)$. In the ambiguity domain, application of the kernel of Cohen's class involves multiplication of the symmetric ambiguity function by the kernel. This

\footnotetext{
3 The ambiguity function reduces to a deterministic correlation function in time if $\theta$ is set to zero. Similarly, as can be seen in a dual form which starts with $Z(\nu)$, it can be seen as a deterministic correlation of spectra if $\tau$ is set to zero.

4 The range of integrals is from $-\infty$ to $\infty$ throughout this paper.
} 
is roughly analogous to application of filters by multiplication of the Fourier transform of the signal by the transfer function in the frequency domain. This operation manifests itself as a convolution in the time domain, thus,

$$
C_{z}(t, \nu, \phi)=F_{\tau}\left[\psi(t, \tau) *_{t} R(t, \tau)\right]
$$

where $\psi(t, \tau)=F_{\theta}^{-1}[\phi(\theta, \tau)]$. Similarly, application of the kernel in the TF domain involves convolution in time and frequency in Cohen's class of distributions. Conventional filtering concepts can be adapted to Reduced Interference Distribution (RID) design.

\subsection{The RID approach}

New distributions with reduced interference (RIDs) [33, 12, 39] have been developed in recent years, serving as attractive alternates for the spectrogram and the WVD. The RID represents an attempt to preserve most of the desirable features of the WVD while alleviating most of the confusing interference between signal components.

RID kernels exhibit low pass characteristics in both the time-shift $(\tau)$ and frequency shift $(\theta)$ dimensions in the ambiguity plane, away from the axes. It has been shown [12, 39 that any real valued symmetric, unit area function $h(t)$ which is limited to insure time and frequency support properties, such that $h(t)=0$ for $|t|>\frac{1}{2}$ and which has a $\left.H(\nu)\right|_{\nu=0}=1$ is a RID kernel. One simply replaces $H(\nu)$ with $H(\theta \tau)$ in order to have a RID kernel in the ambiguity domain form. Usually interference terms are located far from the $\theta-\tau$ origin, so the filter can often be designed to provide strong attenuation of these terms, while preserving the desirable auto-terms of the individual signal components. The form of the RID kernel for the $(t, \tau)$ domain application is $\psi(t, \tau)=\frac{1}{|\tau|} h\left(\frac{t}{\tau}\right)$ and computation is usually most convenient in this domain. Preservation of the time and frequency marginals is one of the important properties of RIDs. One 
can recover the energy spectrum (by integrating with respect to time) or the instantaneous power (by integrating with respect to frequency). This is not the case for spectrograms and many other popular TF distributions (see e.g. [40]).

\section{Application of TF distribution analysis to tur- bulence signals}

A nice discrete kernel which has a binomial form has been used in our applications. This kernel is computationally efficient and also exhibits desirable characteristics in terms of TF distributions [12]. The time frequency distribution obtained by applying the binomial kernel takes the following discrete form:

$$
\begin{aligned}
& \hat{T F_{r i d, b n}}(n, \nu)= \\
& \sum_{\tau=-\infty}^{\tau=\infty} h(\tau) \sum_{v=-|\tau|}^{v=+|\tau|} \frac{g(\nu)}{2^{2|\tau|}}\left(\begin{array}{c}
2|\tau| \\
|\tau|+v
\end{array}\right) z(n+v+\tau) z^{*}(n+v-\tau) e^{-j 4 \pi \nu \tau}
\end{aligned}
$$

where $z[n], n=1 \ldots N$ depicts the sampled time-series under analysis. Functions $h(\tau)$ and $g(\nu)$ are frequency smoothing window and time smoothing window respectively. $h(0)$ and $G(0)=T F_{v}[g(v)]$ are forced to one. Note that these windows were set to $h(\tau)=\operatorname{rect}(\mathrm{N})$ and $g(\nu)=\delta(\nu)$ in the original paper by Williams and Jeong, where $\operatorname{rect}(N)$ stands for the $N$-points rectangular window, and $\delta$ stands for the discrete Dirac distribution. The binomial TFD is a good discrete time approximation of the continuous RID. The binomial TFD (hereafter called the tf-rid) was computed for acoustic signals obtained in the turbulence experiment depicted in the previous sections.

In the cases which are presented, two channels were used to probe the same measurement area : the experimental setup is carefully arranged in such a way

that the measurement areas defined by the intersection of the acoustic beams of each channel are actually superimposed. This latter setting will be maintained for all the experiments presented in the remainder of this paper. First and second channel had the following characteristics $\left(\theta_{s, 1}=60\right.$ degrees, $\left.\nu_{0,1}=20 \mathrm{kHz}\right)$ 
and $\left(\theta_{s, 2}=40\right.$ degrees, $\left.\nu_{0,2}\right)$. Applying eq. (2) and with the transducers arranged as described on figure (1D), one shows that the probed scattering wave vectors are co-axial with the mean flow, and oriented downstream. These latter channel characteristics correspond to $\left|q_{\text {scat }}\right|=369.6 \mathrm{~m}^{-1}$ i.e. the corresponding scale (wavelength) is $1.7 \mathrm{~cm}$, well within the inertial range of the flow. The same wave vectors are probed onto two different channels by tuning $\nu_{0,2}$ such that the condition expressed in eq.(8) is verified. This latter condition is met here for $\nu_{0,2}=29.25 \mathrm{kHz}$. Figure (4) shows the tf-rid obtained for the case where the preceding tuning condition is approximately met (actually, $\nu_{0,2}=29400 \mathrm{~Hz}$ for this experiment). Smoothing windows $h$ and $g$ were both Hanning windows of respective length $L_{h}=129$ and $L_{g}=3$.

The frequency marginals, obtained by integrating the tf-rid over time are shown on the left hand side of the plot. This frequency marginal matches the power density spectrum (PDS) (an approximation is introduced by the use of smoothing windows $h$ and $g$ ). The center of mass $\Delta \nu_{c m}$ of the PSD is indicated. Replacing $\Delta \nu$ by $\Delta \nu_{c m}$ in eq. (44) allows one to estimate the average (estimated over 0.6 seconds) axial mean flow velocity $|\vec{V}| \simeq 5.57 \mathrm{~ms}^{-1}$. This result is in good concordance with alternative measurements of the velocity (hot wire anemometry).

The pertinence of both the tf-rid approach, and the length scale selection, within the context of these experiments is illustrated by the high similarity between the tf-rid obtained from the two different (and independent) measurement channels. Both channels evidence the existence of time localized structures (energy packets in the tf-rid) at the same set of instants and with identical Doppler shifts, which was to be expected as the probed wave vectors are very close to each other (in modulus and direction) and are defined within the same measurement volume (cf figure (11)).

The interest of such tf-rid representation in the present context is thus threefold. First, it emphasizes the fact that the broadening of the averaged spectrum is 
due to both the short duration of the structure or events that are the origin of the scattering effects, and the fluctuations of the Doppler shifts (the center of mass of the energy packets in the tf-rid are not well aligned along an axis $\Delta \nu=$ constant. Second, such representation provides a straightforward way to detect the time of occurrence of events at a given scale. A systematic study of the statistical distributions of such events, as functions of the probed scale is presently a matter of great interest and will be presented in a future paper. Third, the use of two simultaneous channel measurements allows one to test the existence of events at different wavelengths arbitrarily close to each other (scale resolution). In the preceding example, the characteristics were set to probe two close scales (namely $\left|q_{\text {scat }, 1}\right|=369,6 \mathrm{~m}^{-1}$ and $\left|q_{\text {scat }, 2}\right|=371,6 \mathrm{~m}^{-1}$ ). Structures that are evidenced on one channel clearly appear jointly in the second channel; this latter point is illustrated on the central plot of figure (ब), where the normalized time marginal of the geometric mean of the rid of the channels, $d(t)$ is shown:

$$
d(t)=\frac{\int_{f_{n}=0}^{f_{n}=.5} t f r_{r i d, 1}\left(t, f_{n}\right) \cdot t f r_{r i d, 2}\left(t, f_{n}\right) d f_{n}}{\sqrt{\int_{f_{n}=0}^{f_{n}=.5} t f r_{r i d, 1}\left(t, f_{n}\right)} \sqrt{\int_{f_{n}=0}^{f_{n}=.5} t f r_{r i d, 2}\left(t, f_{n}\right)}}
$$

$t f r_{r i d, i}$ is the tf-rid of the signal recorded on channel $i$, and $f_{n}$ is the normalized frequency. Note that $0 \leq d(t) \leq 1$ by virtue of Cauchy-Schwarz inequality. This method (maximum likelihood criterion) allows detection of the joint presence of energy at a given time an d with a given Doppler shift on both channels. Figure (5) allows to evidence the similarity of the signals recorded on the channels, even when considered in details, thus revealing similar detection of the local dynamics.

The same experiment was performed by resetting the analyzing frequency of the second channel to $\nu_{0,2}=35 k H z$. Thus, $\left|\vec{q}_{s c a t, 2}\right|=442,4 m^{-1}$ with the same geometry, i.e. $\vec{q}_{s c a t, 1}$ and $\vec{q}_{s c a t, 2}$ were still colinear, aligned with the flow and oriented downstream. The estimated tf-rids obtained for this situation are 
shown on figure (6). The normalized co-occurrence function $d(t)$ takes lower values than those obtained in the preceding case. Furthermore, the number of co-occurrences that are detected is much lower in this case, compared to the co-occurrence detected when the tuning condition (cf eq. \&) is verified. Further insight is gained by repeating the previous experiments with $\left|q_{\text {scat }, 1}\right|$ held fixed, while $\left|q_{\text {scat }, 2}\right|$ is varied according to $\nu_{0,2}$ ranging from $25 k \mathrm{~Hz}$ to $35 k H z$ (i.e. $\left.316 m^{-1} \leq\left|q_{s c a t, 2}\right| \leq 442 m^{-1}\right)$. For each couple $\left(\vec{q}_{s c a t, 1}, \vec{q}_{s c a t, 2}\right)$, defining a spatial wavector separation $\delta q=\left|\vec{q}_{s c a t, 2}-\vec{q}_{s c a t, 1}\right|$, we compute the RID representations. Then we introduce a time-scale generalized cross-correlation function $C(\tau, \delta q)$ defined as:

$$
C(\tau, \delta q)=\left\langle\frac{\int_{f_{n}=0}^{f_{n}=.5} t f r_{\vec{q}}\left(t, f_{n}\right) \cdot t f r_{\vec{q}+\overrightarrow{\delta q}}\left(t+\tau, f_{n}\right) d f_{n}}{\sqrt{\int_{f_{n}=0}^{f_{n}=.5} t f r_{\vec{q}}\left(t, f_{n}\right)} \sqrt{\int_{f_{n}=0}^{f_{n}=.5} t f r_{\vec{q}+\overrightarrow{\delta q}}\left(t+\tau, f_{n}\right)}}\right\rangle_{t}
$$

where $\langle\bullet\rangle_{t}$ stands for an average over the time variable $t$. Figure (7).a) sketched the results of this series of experiments on a 2D-plot where the amplitude of $C(\tau, \delta q)$ is coded in gray levels, as a function of $\delta q$, and the delay time variable $\tau$. The evolution of $C(\tau, \delta q)$ for 3 values of $\delta q$ (namely $\delta q=0.0,0.2,-0.2$ ) is plotted on Figure (77.b). Figure (7.b and .c) demonstrates that the mean delay time $\tau$ evolves monotonously from negative values when $\delta q \leq 0$ towards positive values when $\delta q \geq 0$. The evolution of the maximum of the correlation function is plotted on figure (7.d) as a function of $\delta q$. As expected, the correlation function is maximum for $\delta q=0$ decreases smoothly as $|\delta q|$ increases. The observed evolutions of both the value and the sign of the delay time is an experimental evidence of a time-continuous turbulent cascade of energy from large scales $(\delta q \leq 0)$ towards smaller scales $(\delta q \geq 0)$. Up to our knowledge, this time continuity of the cascade which is a direct evidence of the time reversal symmetry breaking had not been yet experimentally demonstrated. From Figure (7).b) we measure a typical extension of the detected wave-packets in the q-domain : $\delta q \simeq 0.4 \mathrm{~cm}^{-1}$, corresponding to a spatial correlation length of about $10 \mathrm{~cm}$. It is worth noticing here that the measured correlation length remains smaller 
than the size of our transducers. It is interesting at this point to mention a few words about the spatial and spectral resolutions of the experimental setup. The spatial resolution is determined by the maximum frequency one is able to propagate through the flow of interest; actually, this frequency is around $200 \mathrm{kHz}$ enabling to probe lengthscales as small as $1.5 \mathrm{~mm}$ 26] (this upper frequency limit is due to sound absorption phenomena). The spectral resolution, determining the minimum spatial wavevector separation one can probe, depends directly on the size $L$ of the transducers which fixed the width $\Delta q$ of the equivalent spatial filter 24] ( $\Delta q \simeq \frac{1}{L}$ see also section 2). Notice however, that the latter limitation (analogous to the Rayleigh criterion used in optics) can be overcome by resorting to a deconvolution scheme once the diffraction pattern of each transducer has been modelized or characterized.

\section{Conclusion}

We propose a method for evidencing coherent vorticity structures in turbulent flows. Though this existence seems to be widely accepted and evidenced by numerical simulations [5] or in some peculiar flows [3, 4] it still deserves further experimental efforts. Furthermore, no clear definition seems to exist for coherent structures. The experimental technique described here is derived in the spatial Fourier domain and recovers the intuitive notion of phase stationarity thus allowing one to understand these structures as propagating energy (more precisely enstrophy) packets.

The main advantage of the acoustic technique relies on its global and non pertubative character, as it gives access to the instantaneous value of spatial Fourier components of the vorticity field.

Using two distinct channels (acoustic interferometry) we are able to establish the robustness (noise immunity) of our vorticity detection scheme. In addition, this configuration enables the simultaneous probing of two spatial wave vectors arbitrarily close to each other (scale resolved spectroscopy). This latter 
remark is in contrast with usual one-point measurement (hot wire anemometry) in turbulence which do not allow a clear separation of time and space dynamics. Indeed in such measurements, one has to use the Taylor hypothesis in order to recast time increments into spatial increments.

The joint resort to acoustic scattering and to time-frequency distributions leads to a measurement process whereby time (date of occurrence) and frequency (Doppler shifts) informations are preserved (time resolved spectroscopy). It is worth noticing here that this kind of analysis could be easily transposed to data obtained from numerical simulations. Indeed, in the case of pseudo-spectral direct numerical simulations (DNS), a great part of the computing time is spent in the spatial Fourier space. We claim that time-frequency distributions offer an efficient tool for the detection of coherent structures as it is a "blind" detection : no a priori information is needed (for example on the shape of the structures) and one only resorts to a stationary phase criterion (stating that components of a coherent structure are convected with the same velocity). From the RID representations of the scattered signals (simultaneously acquired), we define a generalized time-scale correlation function based upon a maximum likehood criterion. This time-scale correlation function reveals salient features of the time-scale dynamics of the detected coherent structures. The most interesting one is the existence of a cascade process from larger scale towards smaller one, this process being continuous in time. This latter observation indicates that coherent vorticity structures play a crucial role in the time reversal symmetry breaking, a main feature of the transition to turbulence.

Finaly, we wish to underline here, the similarities between our experimental results and analytical models of coherent structures in the form of spiral vortices recently introduced by T.S. Lundgren 433. We would also like to underline the similarity between our experimental approach, and recent numerical 42 and theoretical [41] approaches relying on wavelet decompositions of the turbulent velocity field (one-point measurement). 


\section{ACKNOWLEDGMENTS}

This work is supported by the Région Rhône-Alpes (Emergence project No.

97027229) and by Pr. J.P. Hansen, member of the Institut Universitaire de France. The authors would like to thank P. Flandrin, ENS-LYON, for stimulating discussions. 


\section{References}

[1] U.Frisch, Turbulence, (Cambridge University Press, 1995).

[2] R. Benzi, S. Ciliberto, C. Baudet \& G. Ruiz Chavarria, " On the scaling of three dimensional homogeneous and isotropic turbulence ", Physica D 80, pp 385-398 (1993).

[3] S.Douady, Y.Couder, M.E.Brachet, " Direct Observations of the intermittency of intense vorticity filaments in turbulence" Phys. Rev. Let. 67, pp 983-986 (1991).

[4] B.Dernoncourt, J.F.Pinton, S.Fauve, "Scaling of vorticity filaments in a turbulent swirling flow " Proceedings of the 6th European turbulence conference, Lausanne, pp 437-440 (1996).

[5] M.E.Brachet, " Direct simulation of three-dimensional turbulence in the Taylor-Green vortex" Fluid. Dyn. Res. 8, pp 1-8 (1991).

[6] M.Lesieur. Turbulence in fluids, 3rd edition, Kluwer, Dordrecht (1997).

[7] Z.S. She \& E. Lévêque, " Universal scaling laws in fully developed turbulence", Phys. Rev. Lett. 72, pp 336-339 (1994).

[8] A.M. Obukhov, "Effect of weak inhomogeneities in the atmosphere on sound and light propagation ", (Izv. Akad. Nauk. Seriya Geofiz. 2 pp 155165 (1953).

[9] R.H.Kraichnan, " The scattering of Sound in a Turbulent Medium " ,J.Acoust. Soc. Am. 25 pp 1096-1104 (1983).

[10] B.T.Chu, L.S.G.Kovàsznay, " Non-linear interactions in a viscous heatconducting compressible gas ", J. Fluid. Mech. 3 pp 494-514 (1958).

[11] F. Lund \& C. Rojas, " Ultrasound as a probe of turbulence ",Physica D 37, pp 508-514 (1989). 
[12] W.J.Williams, J.Jeong, in Time-frequency signal analysis: methods and applications, Ed. B.Bouashash, Longman \& Cheshire, Chapter 3, 74-97 (1992).

[13] G.K.Batchelor, The theory of homogeneous turbulence, Ed. Cambridge University Press, Cambridge (1953).

[14] M.Nelkin, "Universality and scaling in fully developed turbulence", Advances in Physics, 43, pp 143-181 (1994).

[15] A.Noullez, G.Wallace, W.Lempert, R.B.Miles \& U.Frisch, "Transverse velocity increments in turbulent flows using the RELIEF technique", J. Fluid. Mech., 338, pp 287-307 (1997).

[16] J.M.Wallace, " Methods for measuring vorticity in turbulent flows ",Experiments in Fluids, 4, pp 61-71 (1986).

[17] A. Tsinober, E. Kit \& T. Dracos, " Experimental investigation of the field of velocity gradients in turbulent flows ", J. Fluid. Mech., 242, pp 169-192 (1992).

[18] H. S. Shafi \& R. A. Antonia, "Small-scale characteristics of a turbulent boundary layer over a rough wall ", J. Fluid. Mech. 342, pp 263-293 (1997).

[19] T.Colonius, S.K.Lele \& P.Moin, " The scattering of sound waves by a vortex: numerical simulations and analytical solutions "J. Fluid. Mech., Vol.260, pp.271-298 (1994).

[20] R. Engler et al., J.Acoust. Soc. Am. 71 (1), pp $42-50$ (1982), and J.Acoust. Soc. Am. 85 (1) pp.72-82 (1989).

[21] C. M. Ho \& L.S.G. Kovàsznay, "Propagation of a coherent acoustic wave through a turbulent shear flow" ,J.Acoust. Soc. Am. 60 pp 40-45 (1976).

[22] M.S. Korman \& R. T. Beyer, " The scattering of sound by turbulence in water " J.Acoust. Soc. Am. 67 (6) pp 1980-1987 (1976). 
[23] W. Baerg \& W.H. Schwarz, " Measurements of the scattering of sound from turbulence " ,J.Acoust. Soc. Am. 39 (6) pp 1125-1132 (1965).

[24] C.Baudet, S.Ciliberto \& J.F.Pinton, "Spectral analysis of the von Kármán flow using ultrasound scattering ", Phys.Rev.Lett. 67-2, pp 193-195 (1991).

[25] P.R. Gromov, A.B.Ezerskii \& A.L.Fabrikant, "Sound scattering by a vortex wake behind a cylinder ", Sov.Phys.Acoust. 28-6, pp 452-455 (1982).

[26] C.Baudet, R.Hernandez, "Spatial enstrophy spectrum in a fully turbulent jet ", Proceedings of the 6th European turbulence conference, Lausanne, pp 421-424, (1996).

[27] A.S.Monin, A.M.Yaglom, Statistical fluid mechanics, (MIT Press, 1987).

[28] H.Tennekes, J.L.Lumley, A first course in turbulence, (MIT Press, 1972).

[29] D.Anke, Acustica 30, (1974).

[30] L.E.Kinsler, A.R.Frey, A.B.Coppens, J.V.Sanders, Fundamentals of acoustics, (Wiley and Sons, 1982), 3rd edition.

[31] P.M.Morse, K.U.Ingard, Theoretical acoustics, (Princeton University Press, 1986).

[32] W.A.Watkins, Marine Bio-acoustics, 2, 15-43 (1966).

[33] H.I.Choi, W.J.Williams, IEEE Trans. Acoust., Speech, Signal Proc., 37, No. 6, 862-871 (1989).

[34] L.Cohen, Proc. IEEE, 77, No. 7, 941-981 (1989).

[35] L. Cohen, Time-frequency signal analysis, Prentice Hall, 1995.

[36] P.Flandrin, IEEE Int. Conf. Acoust., Speech, Signal Proc., 41B, 4.1-4.4, (1984). 
[37] P.Flandrin, Temps-fréquence, Ed. Hermès, Paris (1993).

[38] F.Hlawatsch,Digital Signal Processing - 84, ed. V.Cappellini and A.Constantinides, 263-367, North-Holland (1984).

[39] J. Jeong, W. J. Williams IEEE Trans. Sig. Proc., 40, No. 11, 27572765(1992).

[40] F.Hlawatsh, G.F.Boudreaux-Bartels, IEEE Signal Processing Magazine, 9, No. 2, 21-67, (1992).

[41] A. Arneodo, E. Bacry, S. Manneville \& J.F. Musy, " Analysis of random cascades using space-scale correlation functions ", Phys. Rev. Lett 80, pp 708-711 (1998).

[42] M. Farge, K. Schneider \& N. K. R. Kevlahan, "Coherent structure eduction in wavelet-forced two-dimensional turbulent flows ", in Dynamics of slender vortices Ed. E. Krause, Cambridge University Press (1998).

[43] T.S. Lundgren, " Strained spiral vortex model for turbulent fine structure ", Phys. Fluids. 25(12), pp 2193-2203 (1982). T.S. Lundgren, " A smallscale turbulence model ", Phys. Fluids. A 5(6), pp 1472-1483 (1993). A.D. Gilbert, "A cascade interpretation of Lundgren's streched spiral vortex model for turbulent fine structure ", Phys. Fluids. A 5(11), pp 2831-2834 (1993). 


\section{FIGURE CAPTIONS}

Figure 1: Experimental setup.

Figure 2: Averaged PDS of direct and differentiated recorded data on a jetflow experiment. The overall length of the data field was $2^{18}$ points . The PDS is estimated from 2048 points data segments with 1024 data points overlap.

Figure 3: (a) Estimated spectra from channels 1 and 2; (b) Estimated spectra of differentiated signals recorded on channels 1 and 2; (c) Estimated crosscoherence function between channels 1 and 2; (d) phase of the cross-channel interspectrum (notice that the frequency span is smaller for this last plot).

Figure 4 channels tf-rid estimation for approximate tuning conditions (only positive values are represented).

Figure 5: 2 channels tf-rid estimation for approximate tuning conditions : details.

Figure 6: 2 channels tf-rid estimation for $\vec{q}_{s c a t, 1}=369,6 m^{-1}$ and $\vec{q}_{s c a t, 2}=$ $442,4 m^{-1}$ (only positive values are represented).

Figure \&: Evolution of the time-scale correlation function $C(\tau, \delta q)$. (a) $2 \mathrm{D}$ plot versus time delay $\tau$ and spatial wavectors separation $\delta q$; (b) Representations of $C(\tau, \delta q)$ for three different values of $\delta q\left(0.0 \mathrm{~cm}^{-1}, 0.2 \mathrm{~cm}^{-1},-0.2 \mathrm{~cm}^{-1}\right)$; (c) Plot of the mean delay time versus $\delta q$; (d) Plot of the maximum of $C(\tau, \delta q)$ versus $\delta q$. 


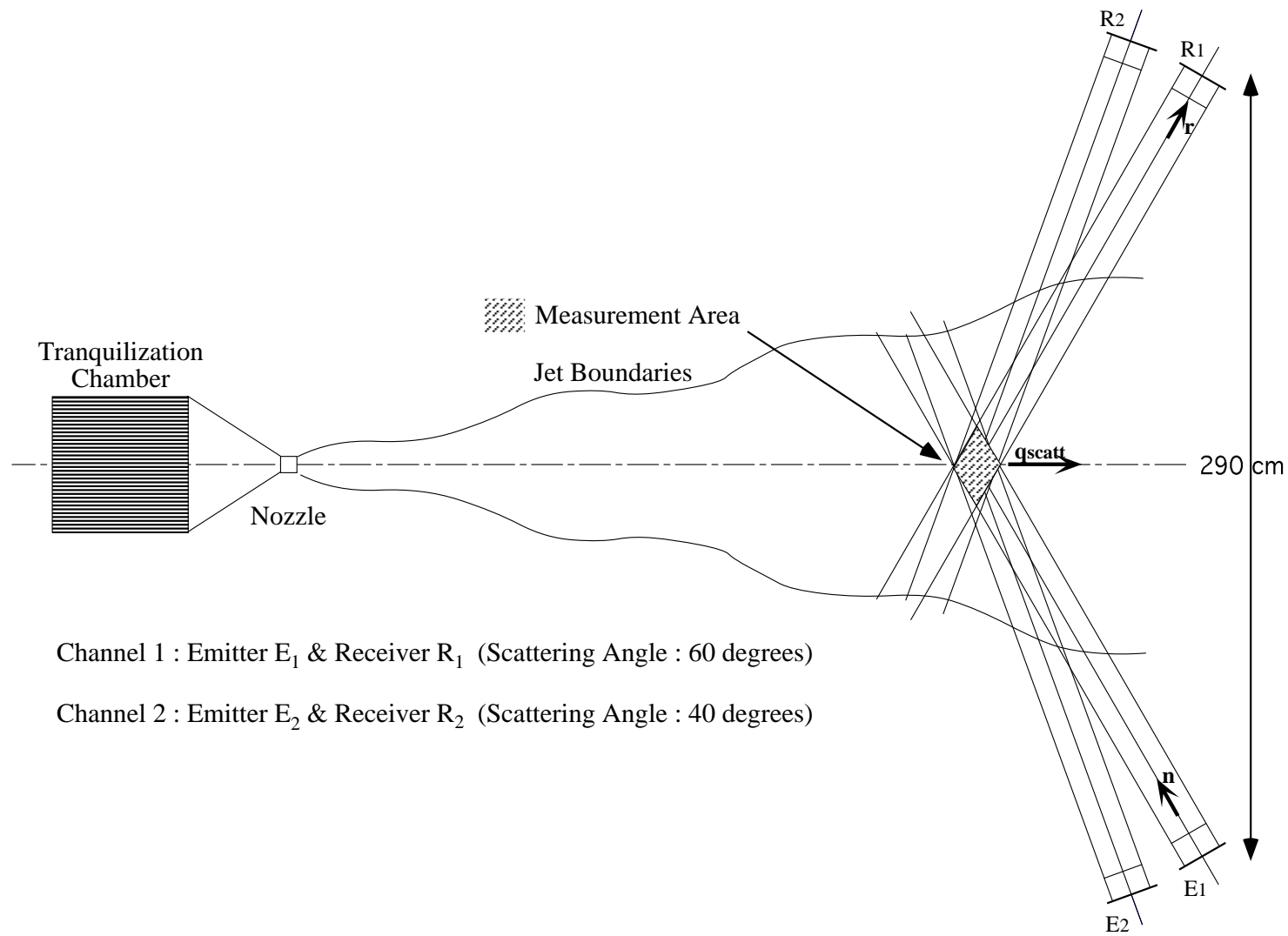

Figure 1: 
(a) Direct

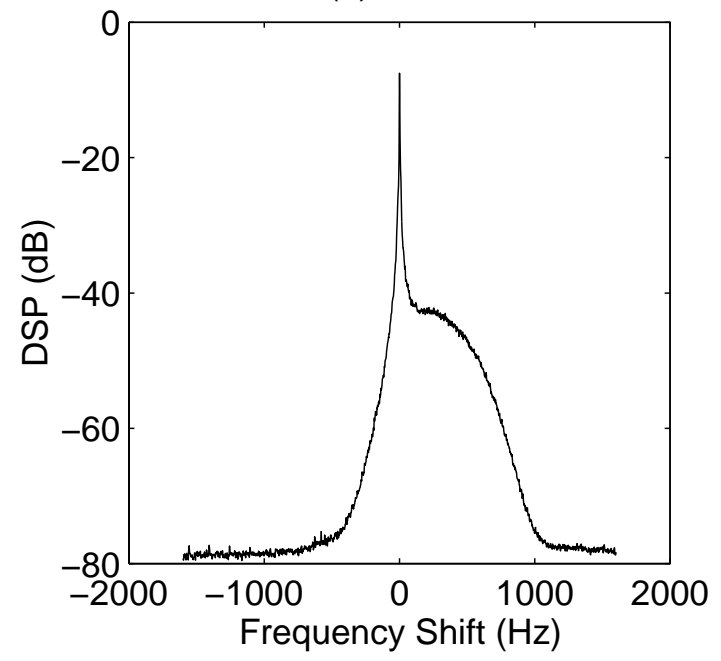

(b) Differenciated

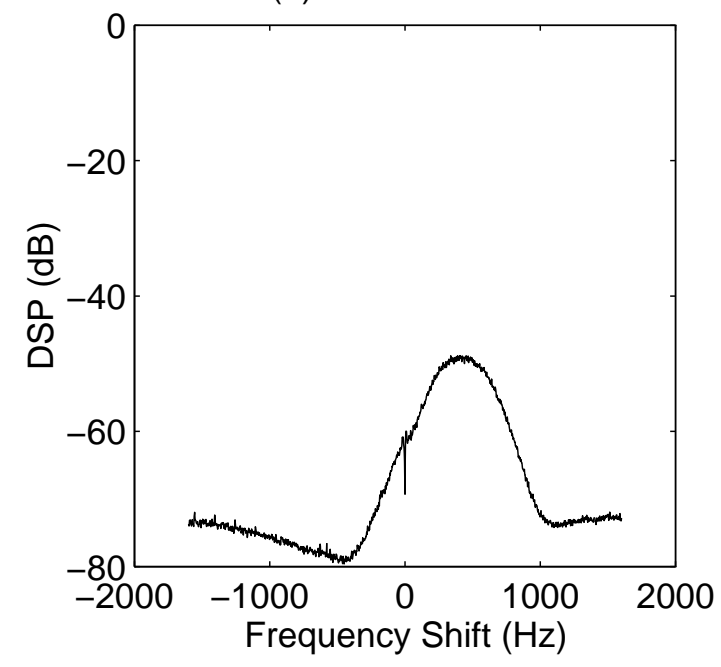

Figure 2: 

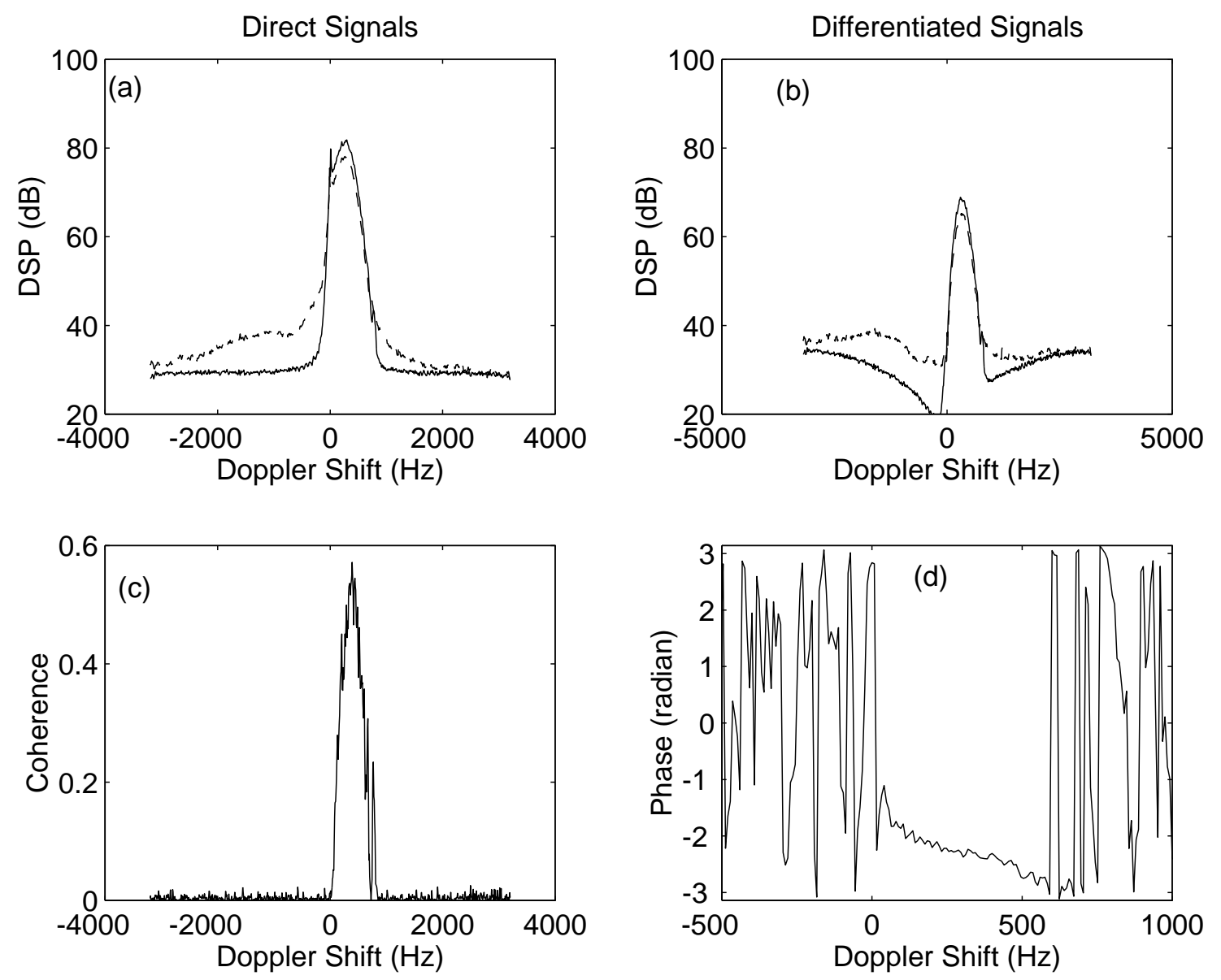

Figure 3: 

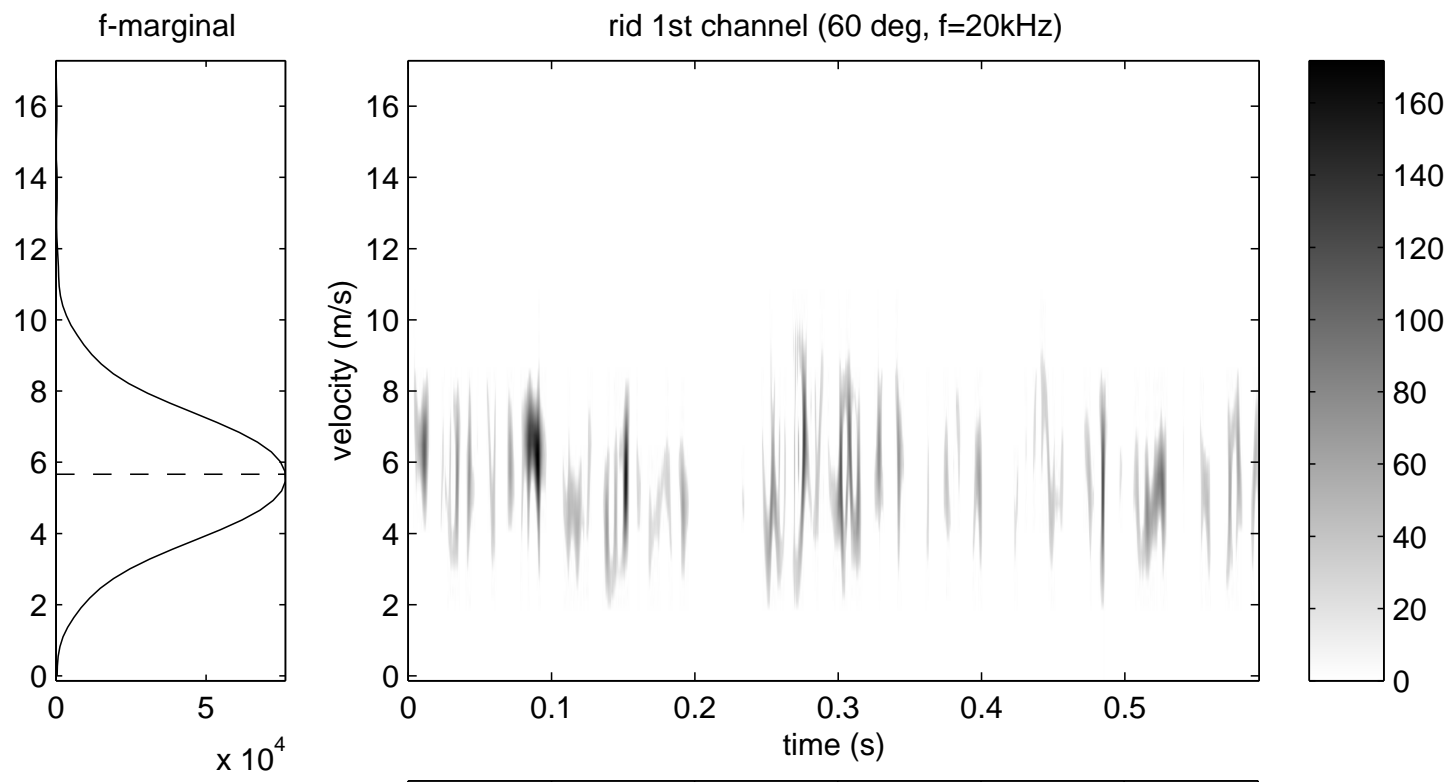

geometric mean ->

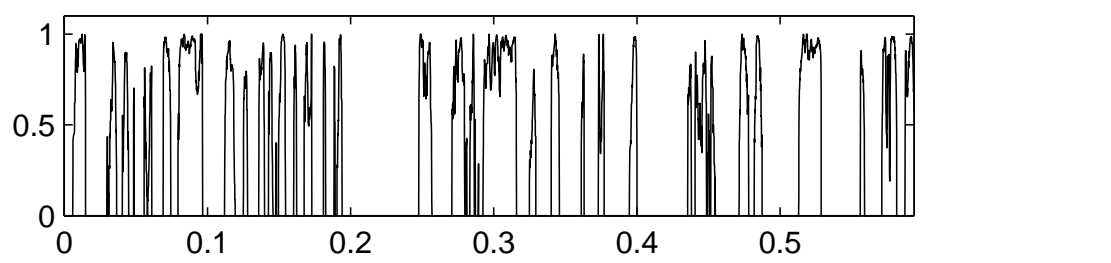

f-marginal

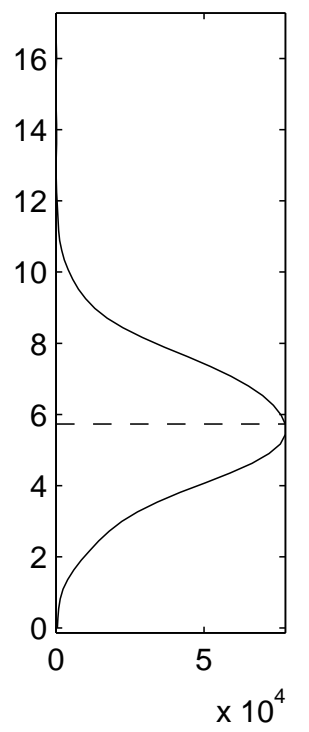

rid 2nd channel (40 deg, $f=29400)$
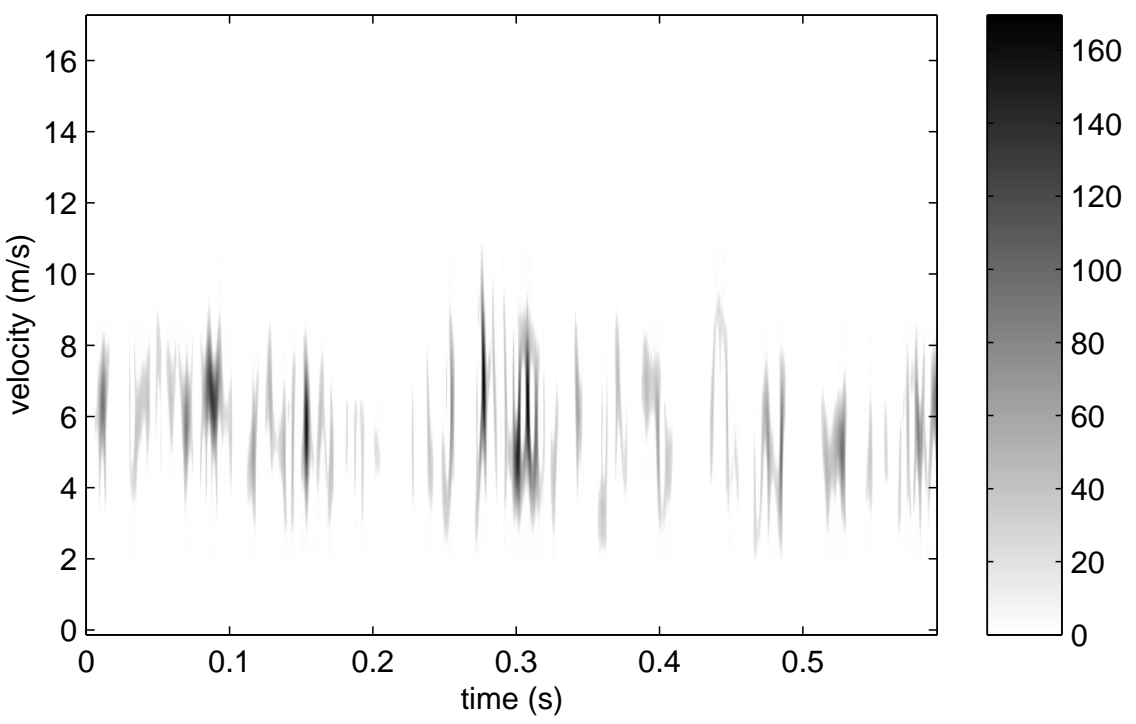

34

Figure 4: 

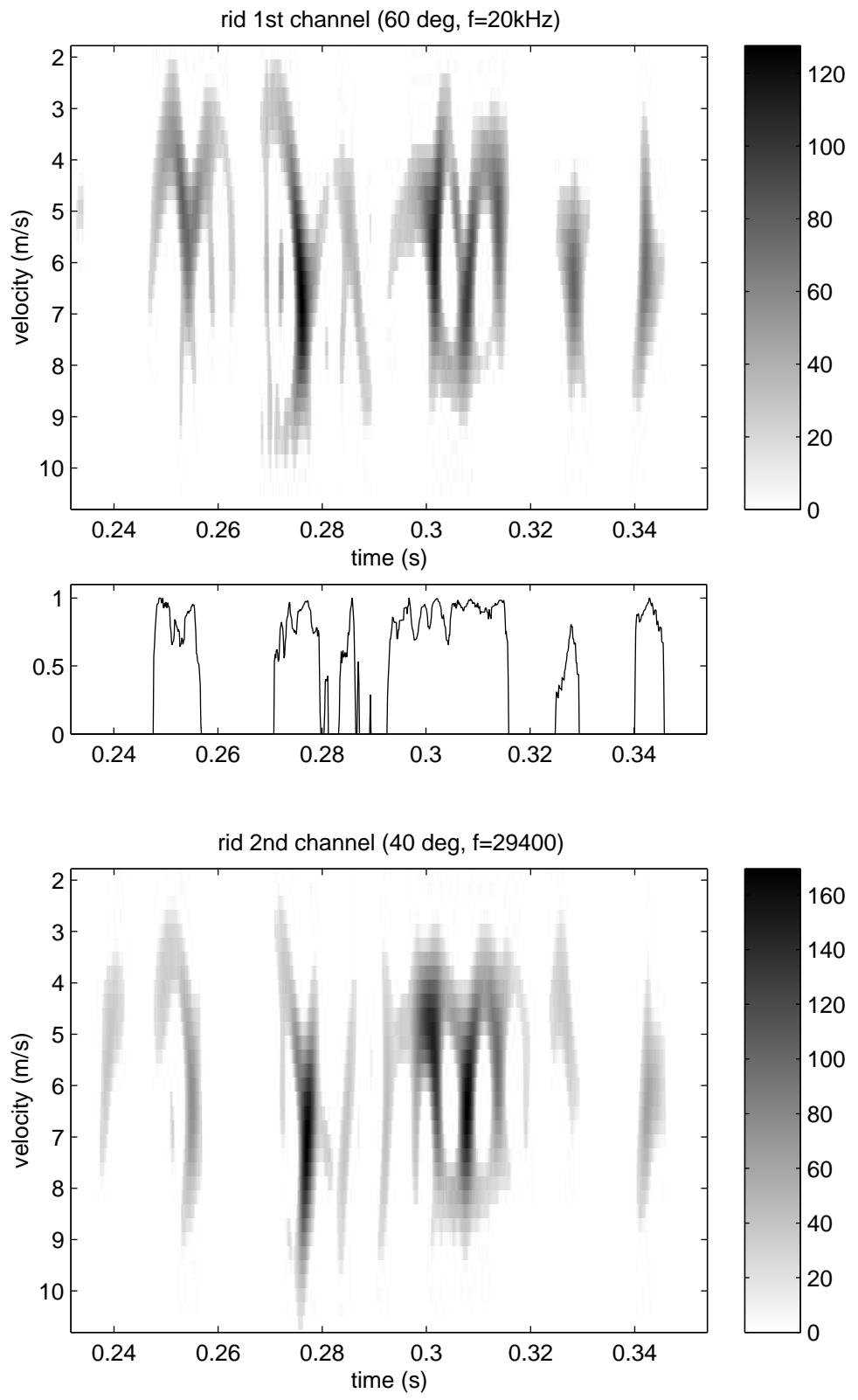

Figure 5: 

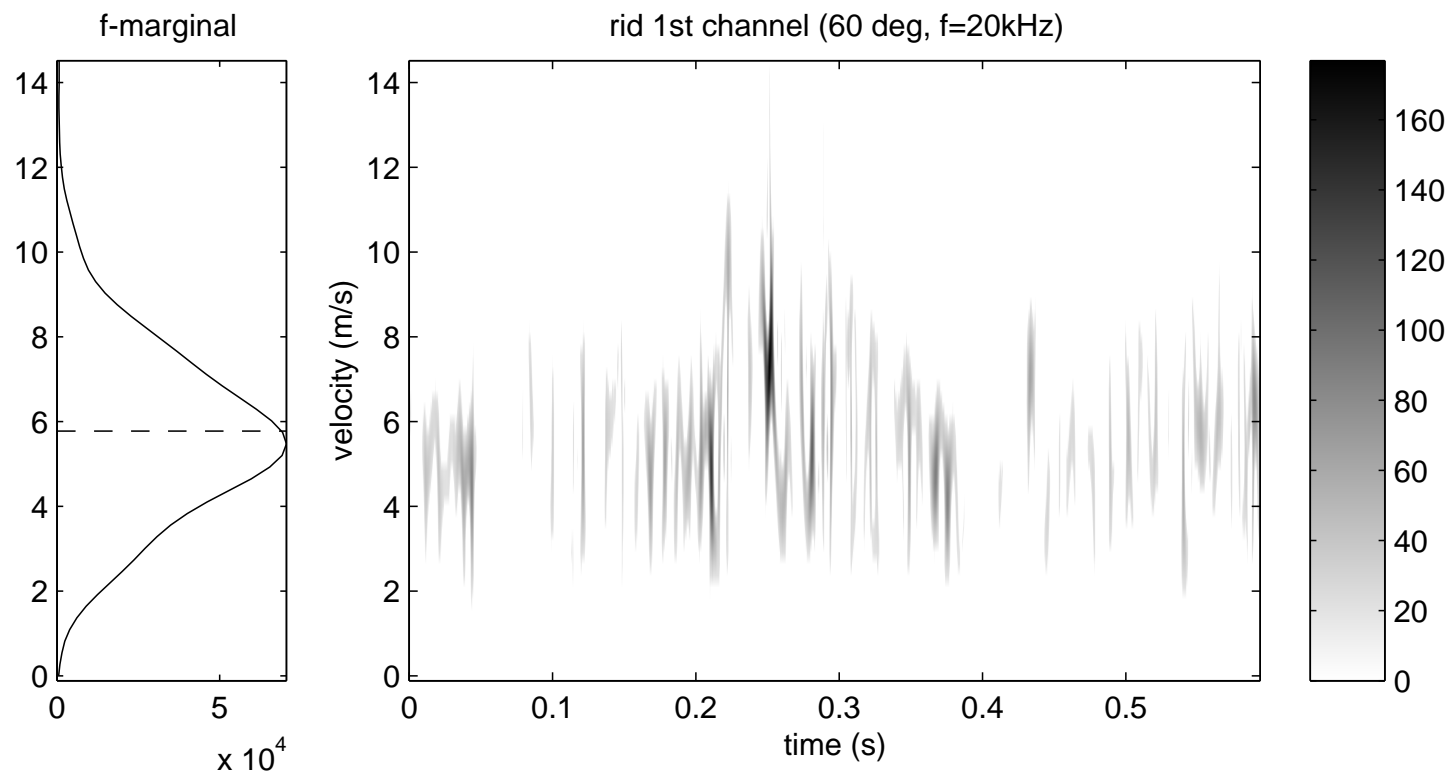

geometric mean ->

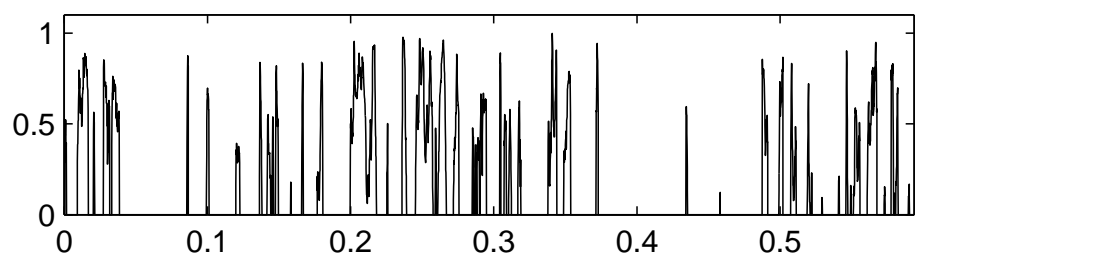

f-marginal

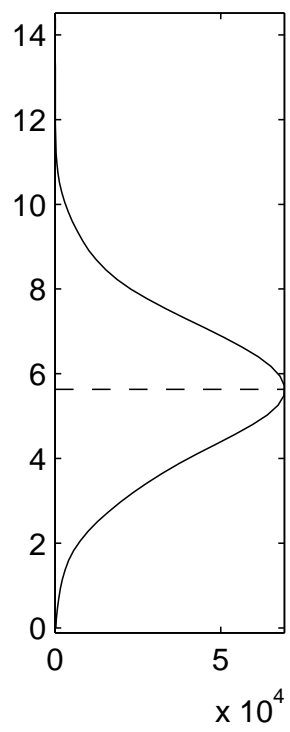

rid 2nd channel (40 deg, $f=35000$ )
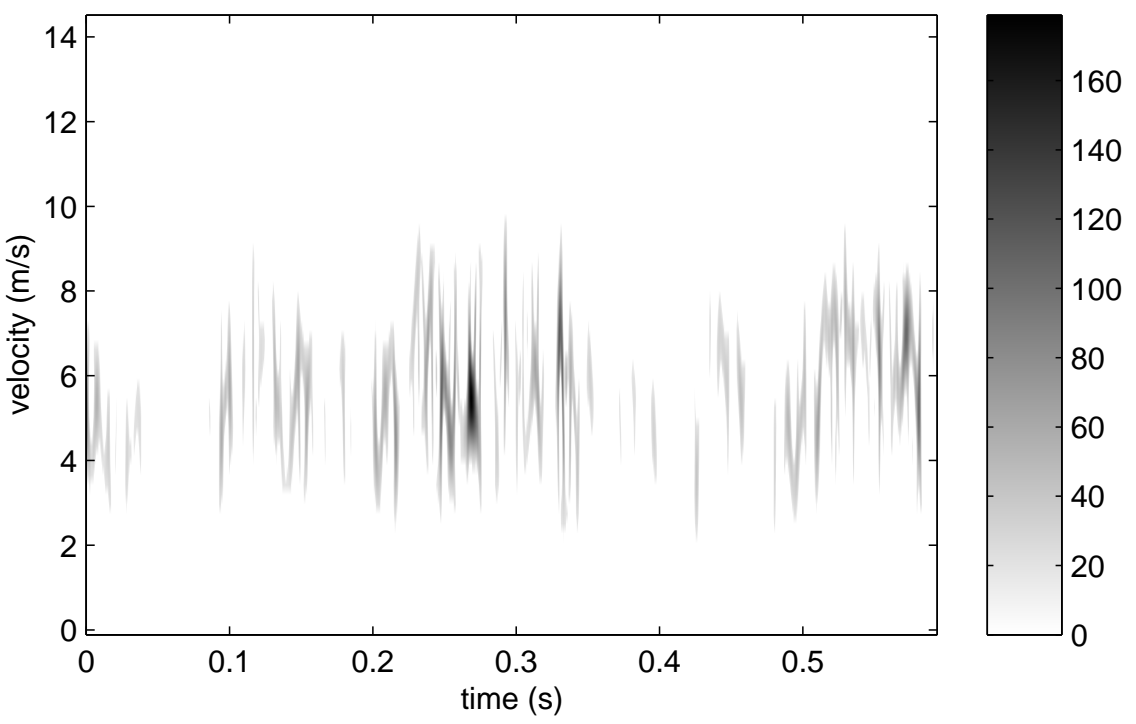

36

Figure 6: 
Figure 7.a

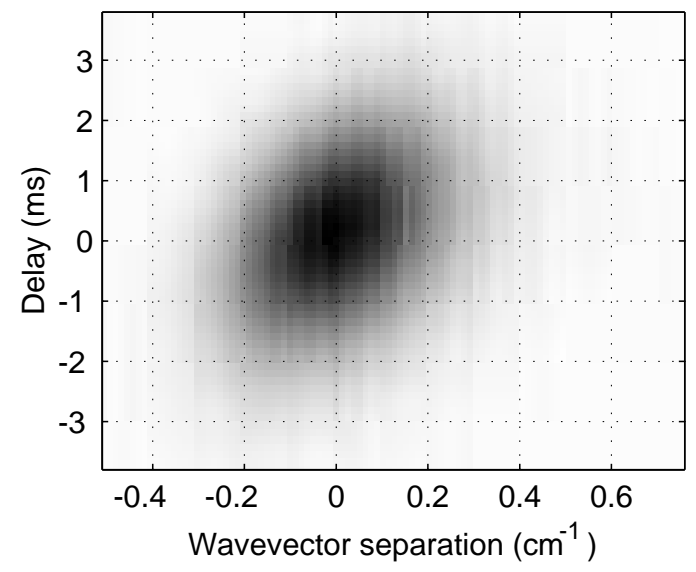

Figure 7.c

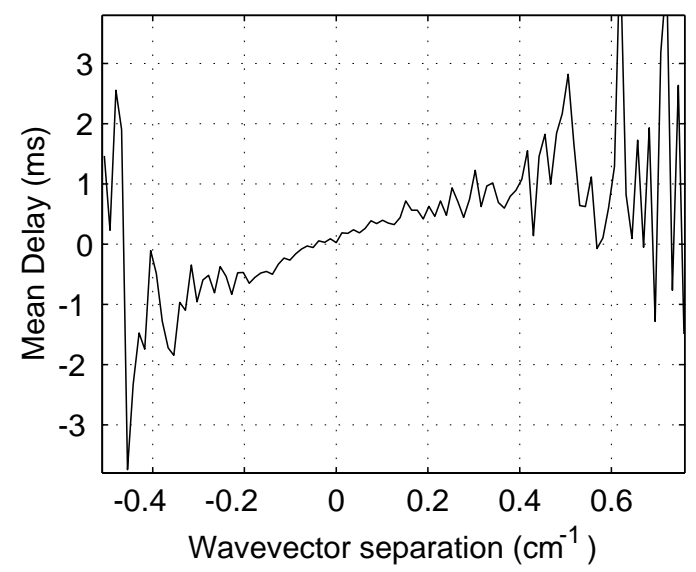

Figure 7.b

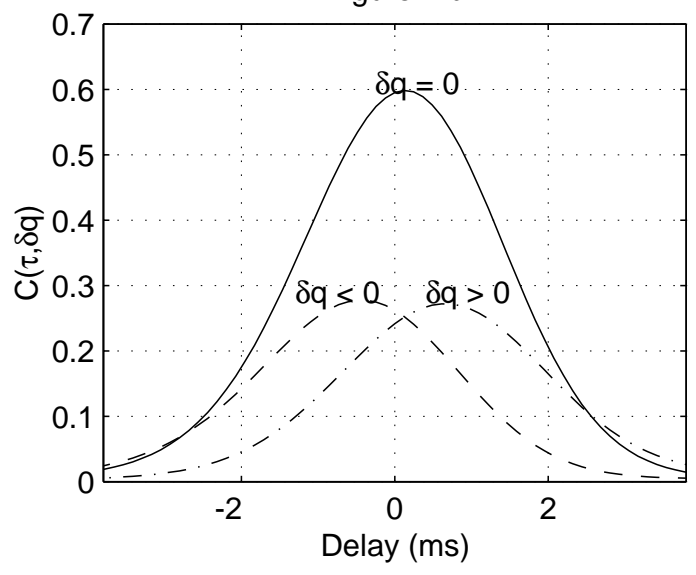

Figure 7.d

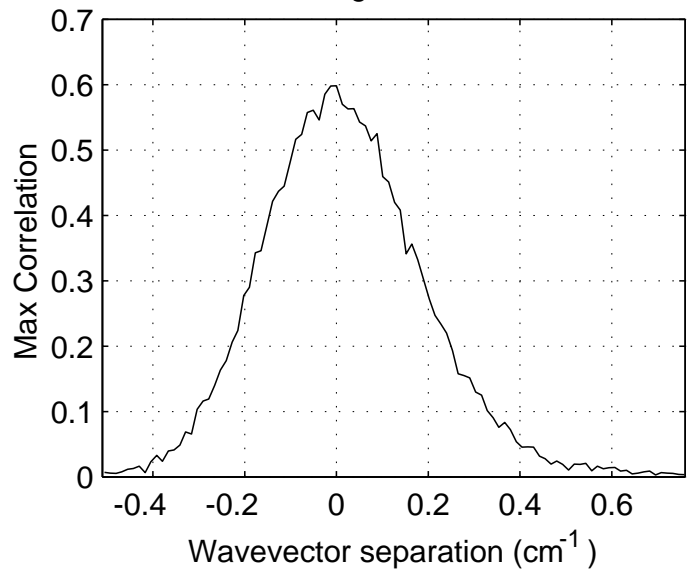

Figure 7: 